\title{
Neighborhood deprivation and availability of culturally specific African-American and Latino fruits and vegetables in five small central Illinois cities
}

\author{
Diana S. Grigsby-Toussaint ${ }^{1^{*}}$, Imelda Kanchule Moise ${ }^{2}$ \\ ${ }^{1}$ Department of Kinesiology and Community Health, University of Illinois at Urbana-Champaign, Champaign, USA; \\ *Corresponding Author: dgrigs1@illinois.edu \\ ${ }^{2}$ Institute of Government and Public Affairs, Center for Prevention Research and Development, University of Illinois at Urbana- \\ Champaign, Champaign, USA
}

Received 13 January 2013; revised 12 March 2013; accepted 14 April 2013

Copyright @ 2013 Diana S. Grigsby-Toussaint, Imelda Kanchule Moise. This is an open access article distributed under the Creative Commons Attribution License, which permits unrestricted use, distribution, and reproduction in any medium, provided the original work is properly cited.

\begin{abstract}
Introduction: Although individual-level dietary behavior among racial/ethnic minority groups in the US is influenced by cultural food preferences and socioeconomic position, few studies of the food store environment have simultaneously examined both factors. The objective of this cross-sectional study was to investigate the availability of culturally specific fruits and vegetables for African Americans and Latinos by levels of neighborhood deprivation. The 5 small central Illinois cities selected for the study have exhibited increasing numbers of both raciall ethnic groups in the last decade. Methods: A validated audit tool was used to survey 118 food stores in 2008. Census 2000 block group data was used to create a neighborhood deprivation index (categorized as low, medium, and high) based on socioeconomic characteristics using principal component analysis. Statistical analyses were performed in SPSS version $\mathbf{1 7 . 0}$ to determine whether the availability of culturally specific fruits and vegetables $(n=31)$ varied by neighborhood levels of deprivation and store type. Results: Fewer than $50 \%$ of neighborhoods carried culturally specific fruits and vegetables, with the lowest availability found in low deprivation neighborhoods $(p<0.05)$. Culturally specific fruits and vegetables were most often found in neighborhoods with medium levels of deprivation, and in grocery stores $(p<0.05)$. Latino
\end{abstract}

fruits and vegetables were less likely to be found across neighborhoods or in stores, compared to African-American fruits and vegetables. Conclusions: The limited availability of culturally specific fruits and vegetables for African Americans and Latinos highlights potential environmental challenges with adherence to daily dietary guidelines for fruit and vegetable consumption in these groups.

Keywords: African-American; Latino; Food Access; Neighborhood; Deprivation; Food Environment;

Food; Culture; Food Store

\section{INTRODUCTION}

Studies exploring the relative contributions of food environment attributes on obesity risk in the US have found that residents of ethnic minority and low income neighborhoods are more likely to experience limited access to food stores as well as limited availability of fruits and vegetables, compared to predominantly White and higher income neighborhoods [1-6]. Moreover, an association has been found between food purchasing preferences and cultural context that influences habitual dietary patterns for racial and ethnic minority groups in the US [7-9]. Yeh et al. 2008, explored barriers to fruit and vegetable consumption in the US and found that Latino immigrants were less likely to purchase fruits and vegetables that were unfamiliar in appearance, based on their country of origin [10]. Another study by Ard et al. 2007 draws our attention to the importance of culturally relevant 
fruits and vegetables among racial minority populations [11]. The researchers found that although culturally relevant fruits and vegetables such as collard greens and okra were more costly than other commonly consumed fruits and vegetables, they were more likely to be found in the homes of African-American families with school children. Notwithstanding the insights gained from these studies, there remains a paucity of food environment studies specifically exploring fruit and vegetable varieties that may be favored by ethnic or racial minority populations in the US $[1,12,13]$. This omission in the study of food environments may limit our ability to implement effective population-based policies, as we may not be documenting a critical factor influencing purchasing behaviors and subsequent dietary patterns, particularly for racial and ethnic minority groups [5]. In addition, these aforementioned studies have focused on either major metropolitan areas, with little work of this type on small metropolitan areas in the US.

Specifically, most studies have focused primarily on either major metropolitan areas such as Chicago [1] or Detroit [14] or rural areas [15]. Recent US census estimates indicate, however, that there are growing numbers of racial/ethnic minority groups in smaller cities in the US [16]. The cities included as part of our study, for example, experienced major increases in African-American and Latino populations between 2000 and 2010 [17]. In Rantoul, IL, one of the cities included in our survey, the population of African-Americans increased from $16.88 \%$ in 2000 to $22.72 \%$ in 2010 , while the percentage of Latinos increased from $2.69 \%$ to $9.67 \%$ during the same time frame [17]. Consequently, such cities represent an ideal setting to explore the availability of culturally relevant fruits and vegetables, which may be critical for long-term public health planning if these populations continue to grow at their current rate.

The objective of our study was to examine the availability of culturally specific fruits and vegetables for African-Americans and Latinos by levels of neighborhood deprivation across 5 cities in central Illinois. We also examined whether differences existed across levels of neighborhood deprivation by store type.

\section{METHODS}

\subsection{Setting}

Five cities located in central Illinois, Champaign, Danville, Decatur, Rantoul, and Urbana, were selected as the target areas for this study. In addition to meeting the definition of small metropolitan areas [18], these cities were also part of the sampling frame for a larger research project on childhood obesity undertaken in conjunction with this study [19]. In 2010, the average concentration of African Americans and Latinos in these cities was
$20.9 \%$ and $6 \%$ respectively [17]. Individual poverty rates across these cities are twice the US average (e.g., 26.9\% compared to the US at $13.1 \%$ ).

\subsection{Sample}

To draw a sample of stores to survey, North American Industry Classification System (NAICS) codes were used to identify 143 stores from a purchased InfoUSA database. However, only 118 stores were included in the final analysis due to refusals $(\mathrm{N}=9)$ or stores not meeting our definitions based on field observations $(\mathrm{N}=16)$. Stores were classified either as grocery stores (i.e., stores with both fresh produce and meat sections) or convenience/ corner stores for data analysis.

\subsection{Deprivation Index}

Building on the work of Sharkey et al. (2010) [15] and US census 2000 data from Summary File 3 was used to create a neighborhood [(defined as census block groups $(\mathrm{n}=79)$ ] deprivation index. A principle component analysis was conducted in STATA version 11.0 with variables z-standardized. The variables used included unemployment, poverty, low education attainment (e.g., less than a 10th grade education), household crowding (e.g., occupied households with more than 1 person per room), households receiving public assistance, households without a vehicle, and households without a telephone. A raw deprivation index was first calculated by multiplying each variable with a factor loading, and then standardized by dividing the eigenvalue of the component (mean $=0$, standard deviation $=1$ ). Based on the distribution of scores for the index, a three-category variable for overall neighborhood socioeconomic deprivation was constructed and assigned to each census block group using the same categorizations of Sharkey et al. (2010) [15]: low, medium and high deprivation corresponding to the lowest, middle and highest quartiles of deprivation scores respectively. Audited stores were then assigned to low, medium or high deprivation neighborhoods using ArcGIS version 9.3 (Redlands, CA: Environmental Systems Research Institute, Inc., 1999).

\subsection{Audit Instrument and Data Collection Procedures}

In order to assess food availability and accessibility, we utilized a validated audit tool adapted from another study in Chicago, Illinois [1,2]. The instrument assesses the availability of commonly consumed (e.g., apples) and culturally-specific fruits and vegetables for African Americans (e.g., black eye peas) and Latinos (e.g., cherimoya) [1,2]. Prior to data collection, store owners and managers were mailed letters explaining the purpose 
of the study, and provided the option to contact the Principal Investigator with questions or to decline participation. The inter-rater reliability (0.81) of the audit instrument was assessed by trained graduate students at various types of food stores before fieldwork. In total, we assessed the respective availability (presence or absence) of 13 and 18 fruits and vegetables preferred by African American and Latino communities. This categorization was based on a review of the existing literature on cultural food patterns among African-Americans and Latinos, as well as existing instruments [1,20-23].

\subsection{Data Analysis}

The availability of each of the 31 fruit and vegetable varieties across all food stores, by neighborhood level of deprivation (i.e., low, medium, high) was assessed using descriptive statistics (e.g., frequencies). Chi-square tests or Fisher's exact tests were used to test for differences in the availability of fruits and vegetables by levels of neighborhood deprivation and store type. Statistical analyses were performed with SPSS 17.0 (IBM Corporation, Chicago, Illinois), with $p<0.05$ considered as significant. The Institutional Review Board at the University of Illinois at Urbana-Champaign designated the procedures for this study as exempt.

\section{RESULTS}

\subsection{Neighborhood Characteristics and Store Distribution}

The study sample consisted of 85 convenience stores and 33 grocery stores. The distribution of stores was similar across levels of neighborhood deprivation, with 40 stores in low deprivation neighborhoods, and 39 stores each in medium and high deprivation neighborhoods respectively. Based on US Census 2000 estimates, the average poverty rate across neighborhoods in our study was $19 \%$, although high deprivation neighborhoods had a poverty rate of $34 \%$. African American and Latinos were most likely to reside in high deprivation neighborhoods (approximately 37\% for both groups), while low deprivation neighborhoods were predominately White (approximately 91\%).

\subsection{Culturally Specific Fresh Fruits and Vegetables by Levels of Neighborhood Deprivation}

Table 1 shows significant differences in availability of certain types of culturally specific fresh fruits and vegetables. Fewer than $50 \%$ of stores across all levels of deprivation carried any African-American culturally relevant fruits or vegetables. Sweet potatoes (34.7\%) were the most commonly available item, while chard (3.4\%) was the least available item across all neighborhoods. Compared to stores in low and high deprivation neighborhoods, stores located in medium deprivation neighborhoods were more likely to carry beets (28\%), chard (10.3\%), collard greens (28.2\%), kale (23\%), and turnip greens $(28.2 \%)(p<0.05)$. Low deprivation neighborhoods, however, were more likely to carry green peas ( $p$ $<0.05)$.

For culturally specific Latino fruits and vegetables, pineapple was the only item that was available in more than $50 \%$ of stores across all levels of deprivation. The only significant difference in availability across levels of deprivation was for tomatillos, where high deprivation neighborhoods were most likely to carry the item (15.4\%). Although not significant, high deprivation neighborhoods were also more likely to carry pineapple (59\%) and chili peppers (15.4\%). Cactus, guava, and passion fruit were unavailable across all stores.

\subsection{Culturally Specific Fruits and Vegetables by Store Type}

Table 2 shows the distribution of culturally-specific fruits and vegetables by store type. For African-American culturally relevant fruits and vegetables, at least 6 items, namely, sweet potatoes (72.7\%), green peas (72.7\%), spinach (63.6\%), black eye peas (69.7\%), kidney beans (69.7\%), and pinto beans (66.7\%) were carried by more than $50 \%$ of grocery stores. Convenience stores were more likely to carry various types of beans or peas, while chard was the least available across both groceries (12.1\%) and convenience stores (0.0\%). Significant differences in availability across grocery and convenience stores were found for all African-American items.

Similar to African-American culturally-relevant fruits and vegetables, 5 Latino items were carried by more than $50 \%$ of grocery stores, namely avocado (63.6\%), black beans $(90.2 \%)$, garbanzo beans (54.5\%), mangoes (57.6\%) and pineapple (75.8\%). Significant differences in the availability of avocado, coconut, mangoes, papaya, pineapple, plantains, chili peppers, jicama, chayote squash, tomatillos, cassava, black beans and garbanzo beans were found by store type.

\section{DISCUSSION}

Overall, our results suggest several similarities, but also several differences in the availability of culturally relevant fruits and vegetables across levels of neighborhood deprivation and store type. In the current study, high deprivation neighborhoods tended to have comparable or better availability of fruits and vegetables that are typically preferred by African-Americans and Latinos, compared to low deprivation neighborhoods. Although these results differ from some published studies [3,15, 
Table 1. Availability of culturally specific fruits and vegetables by neighborhood deprivation, 5 central Illinois cities, 2008.

\begin{tabular}{|c|c|c|c|c|}
\hline \multicolumn{5}{|c|}{ African Americans' culturally specific fresh fruits and vegetables } \\
\hline \multirow{2}{*}{ Item } & \multicolumn{4}{|c|}{ Levels of Deprivation across surveyed stores } \\
\hline & All $(n=118) \%(N)$ & Low $(n=40) \%(N)$ & Medium (n = 39) \% (N) & High $(n=39) \%(N)$ \\
\hline Acorn Squash & $15.3(18)$ & $7.5(3)$ & $25.6(10)$ & $12.8(5)$ \\
\hline Beets & $16.1(19)$ & $5.0(2)$ & $28.2(11)$ & $15.4(6)^{*}$ \\
\hline Black Eye Peas ${ }^{\mathrm{a}}$ & $30.5(36)$ & $27.5(11)$ & $38.5(15)$ & $25.6(10)$ \\
\hline Chard & $3.4(4)$ & $0.0(0)$ & $10.3(4)$ & $0.0(0)$ \\
\hline Collard Greens & $16.9(20)$ & $7.5(3)$ & $28.2(11)$ & $20.5(3)^{*}$ \\
\hline Kale & $12.7(15)$ & $5.0(2)$ & $23.1(9)$ & $10.3(4)^{*}$ \\
\hline Kidney Beans ${ }^{\mathrm{a}}$ & $33.9(40)$ & $32.5(13)$ & 38.5 (15) & $30.8(12)$ \\
\hline Green Peas & $8.5(10)$ & $7.5(3)$ & $7.7(3)$ & $10.3(4)$ \\
\hline Green Peas & $8.5(10)$ & $7.5(3)$ & $7.7(3)$ & $10.3(4)^{*}$ \\
\hline Mustard Greens & $16.9(20)$ & $7.5(3)$ & $25.6(10)$ & $17.9(7)$ \\
\hline Okra & $17.8(21)$ & $12.5(5)$ & $23.1(9)$ & $17.9(7)$ \\
\hline Pinto Beans ${ }^{\mathrm{a}}$ & 33.1 (39) & $22.5(9)$ & $46.2(18)$ & $30.8(12)$ \\
\hline Spinach & $23.6(31)$ & $22.5(9)$ & $25.6(10)$ & $30.8(12)$ \\
\hline Sweet Potatoes & $34.7(41)$ & $27.5(11)$ & 38.5 (15) & $38.5(15)$ \\
\hline Turnip Greens & $17.8(21)$ & $7.5(3)$ & $28.2(11)$ & $17.9(7)^{*}$ \\
\hline \multicolumn{5}{|c|}{ Latino culturally specific fresh fruits and vegetables } \\
\hline Avocado & $20.3(24)$ & $12.5(5)$ & $25.6(10)$ & $23.1(9)$ \\
\hline Black Beans $^{\mathrm{a}}$ & $20.3(24)$ & $15.0(6)$ & $28.2(11)$ & $17.9(9)$ \\
\hline Cactus & $0.0(0)$ & $0.0(0)$ & $0.0(0)$ & $0.0(0)$ \\
\hline Cassava & $1.7(2)$ & $0.0(0)$ & $5.1(2)$ & $0.0(0)$ \\
\hline Chayote Squash & $4.2(5)$ & $0.0(0)$ & $7.7(3)$ & $5.1(2)$ \\
\hline Cherimoya & $0.8(1)$ & $0.0(0)$ & $0.0(0)$ & $2.6(1)$ \\
\hline Chili Peppers & $10.2(12)$ & $7.5(3)$ & $7.7(3)$ & $15.4(6)$ \\
\hline Coconut & $11.9(11)$ & $7.5(3)$ & $17.9(7)$ & $10.3(4)$ \\
\hline Garbanzo Beans $^{\mathrm{a}}$ & $19.5(23)$ & $10.0(4)$ & $25.6(10)$ & $23.1(9)$ \\
\hline Guava & $0.0(0)$ & $0.0(0)$ & $0.0(0)$ & $0.0(0)$ \\
\hline Jicama & $4.2(5)$ & $0.0(0)$ & $7.7(3)$ & $5.1(2)$ \\
\hline Passion Fruit & $0.0(0)$ & $0.0(0)$ & $0.0(0)$ & $0.0(0)$ \\
\hline Mangoes & $20.3(24)$ & $12.5(5)$ & $28.2(11)$ & $20.5(8)$ \\
\hline Papaya & $5.9(7)$ & $2.5(1)$ & $10.3(4)$ & $5.1(2)$ \\
\hline Pineapple & $50.8(60)$ & $50.0(20)$ & $43.6(17)$ & $59.0(23)$ \\
\hline Plantains & $9.3(11)$ & $5.0(2)$ & $15.4(6)$ & $7.7(3)$ \\
\hline Tomatillos & $10.2(12)$ & $0.0(0)$ & $15.4(6)$ & $15.4(6)$ \\
\hline
\end{tabular}

Values calculated for all stores surveyed in the study area $(\mathrm{n}=118)$. Chi square tests or Fisher's exact tests were used to assess differences in availability. ${ }^{*} p<$ 0.05. N = number of surveyed stores that carried item. Questions assessed availability of African American and Latino commonly consumed fruits and vegeta-

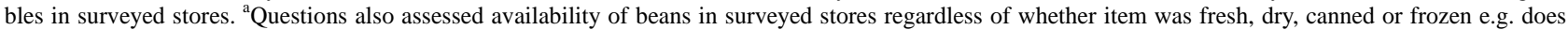
the store carry black beans? 
Table 2. Availability of culturally specific fruits and vegetables by store type, 5 central Illinois cities, 2008.

\begin{tabular}{|c|c|c|}
\hline \multicolumn{3}{|c|}{ African Americans' culturally specific fruits and vegetables } \\
\hline Item & $\begin{array}{c}\text { Grocery Stores } \\
(\mathbf{n}=33)\end{array}$ & $\begin{array}{c}\text { Convenience Stores } \\
(\mathbf{n}=\mathbf{8 5})\end{array}$ \\
\hline & $\%(N)$ & $\%(\mathrm{~N})$ \\
\hline Acorn Squash & $45.5(15)$ & $3.5(3)^{* * *}$ \\
\hline Beets & $48.5(16)$ & $3.5(3)^{* * *}$ \\
\hline Black Beans $^{\mathrm{a}}$ & $90.2(22)$ & $8.3(2)^{* * *}$ \\
\hline Black Eye Peas ${ }^{\mathrm{a}}$ & $69.7(23)$ & $15.3(13)^{* * * *}$ \\
\hline Chard & $12.1(4)$ & $0.0(0)^{* *}$ \\
\hline Collard Greens & $48.5(16)$ & $7.1(6)^{* * *}$ \\
\hline Green Peas & $72.7(24)$ & $45.9(39)^{*}$ \\
\hline Kale & $42.4(14)$ & $1.2(1)^{* * * *}$ \\
\hline Kidney Beans ${ }^{\mathrm{a}}$ & $69.7(23)$ & $20.0(17)^{* * *}$ \\
\hline Mustard Greens & $48.5(16)$ & $4.7(4)^{* * *}$ \\
\hline Okra & $45.5(15)$ & $7.1(6)^{* * *}$ \\
\hline Pinto Beans ${ }^{a}$ & $66.7(22)$ & $20.0(17)^{* * *}$ \\
\hline Spinach & $63.6(21)$ & $11.8(10)^{* * *}$ \\
\hline Sweet Potatoes & $72.7(24)$ & $20.0(17)^{* * *}$ \\
\hline Turnip Greens & $42.4(14)$ & $8.2(7)^{* * *}$ \\
\hline \multicolumn{3}{|c|}{ Latino culturally-specific fruits and vegetables } \\
\hline Avocado & $63.6(21)$ & $4.7(4)^{* * * *}$ \\
\hline Black Beans $^{\mathrm{a}}$ & $57.6(19)$ & $5.9(5)^{* * * *}$ \\
\hline Cassava & $6.1(2)$ & $0.0(0)^{*}$ \\
\hline Cactus & $0.0(0)$ & $0.0(0)$ \\
\hline Chayote Squash & $12.1(4)$ & $1.2(1)^{*}$ \\
\hline Cherimoya & $3.0(1)$ & $0.0(0)$ \\
\hline Chili Peppers & $27.3(9)$ & $3.5(3)^{* * *}$ \\
\hline Coconut & $30.3(10)$ & $4.7(4)^{* * *}$ \\
\hline Garbanzo Beans $^{\mathrm{a}}$ & $54.5(18)$ & $5.9(5)^{* * * *}$ \\
\hline Guava & $0.0(0)$ & $0.0(0)$ \\
\hline Jicama & $15.2(5)$ & $0.0(0)^{* * *}$ \\
\hline Mangoes & $57.6(19)$ & $5.9(5)^{* * * *}$ \\
\hline Passion Fruit & $0.0(0)$ & $0.0(0)$ \\
\hline Papaya & $15.2(5)$ & $2.4(2)^{*}$ \\
\hline Pineapple & $75.8(25)$ & $41.2(35)^{* *}$ \\
\hline Plantains & $27.3(9)$ & $2.4(2)^{* * * *}$ \\
\hline Tomatillos & $27.3(9)$ & $3.5(3)^{* * *}$ \\
\hline
\end{tabular}

Values calculated for all stores surveyed in the study area $(\mathrm{n}=118)$. $\mathrm{N}=$ number of surveyed stores that carried item. Questions assessed availability of African American and Latino commonly consumed fruits and vegetables in surveyed stores regardless of whether item was fresh, dry, canned or frozen e.g. does the store carry black beans? Chi-square test statistics $={ }^{* * * *} p$ $=0.000 ;{ }^{* * *} p=0.001 ;{ }^{*} p=0.01$.
24], they are consistent with those of Smith and colleagues (2010) who found that neighborhoods with the highest levels of deprivation also had the best availability of fruits and vegetables [25]. The results are also in accord with a recent study, which showed higher availability of food stores in low income neighborhoods than was previously thought [26]. Another possible explanation for this may be the presence of fewer grocery food stores (data not shown) in low deprivation neighborhoods compared to medium and high deprived neighborhoods in our study area.

Our results are significant in at least two major respects. First, the limited availability of food stores with culturally-relevant items in high deprivation neighborhoods still remains a concern. Second, according to the US 2010 Census, high deprivation neighborhoods in the current study area have higher concentrations of African-Americans and Latinos, where limited availability of culturally specific fruits and vegetables may impact adherence to recommended daily dietary intakes of fruits and vegetables among these groups [17]. This is of particular concern, as the five cities included in this study together experienced sharp demographic shifts among African-Americans and Latinos between 2000 and 2010, with the cities of Champaign and Rantoul experiencing the most growth in the Latino population. The Latino population in the city of Champaign was $6.31 \%$, rising to 5111 people in 2010 from 2724 in 2000 and it tripled in Rantoul to $9.7 \%$ of the population between 2000 and 2010 [17]. African Americans also make up between $15.6 \%$ (Champaign) to $30.2 \%$ (Danville) of the city populations in the study area [17].

Another important finding was that grocery stores had better availability of culturally specific fruits and vegetables compared to convenience stores. This result corroborates the findings of several studies examining the neighborhood food environment $[1,13]$. Still, few grocery stores carried more than $50 \%$ of the culturally specific fruits and vegetables assessed. Moreover, convenience stores which were more likely to be found in the high deprivation neighborhoods where most African Americans and Latinos live were also less likely to carry culturally specific fruits and vegetables, thus, barriers to dietary consumption for both African Americans and Latinos remain a concern.

This study is not without limitations. We did not examine other aspects of the food environment that might influence purchasing behaviors such as price or selection. We also did not use dietary recall to determine which items were most often consumed by the African-Americans and Latinos in our area, so we may also have excluded items of relevance. Consequently, we may have under or overestimated the availability of culturally specific fruits and vegetables for both groups in our study 
area.

The strengths of this study, however, lie in the setting and design. The 5 small cities in central Illinois represent an understudied area for examining inequalities in the food environment for African Americans and Latinos in the US, where major metropolitan and rural areas have typically been the focus of most studies [27]. Both statistical and geospatial techniques were used to create a neighborhood index to assess availability of culturally relevant fruits and vegetables by neighborhood deprivation and to assign stores to their designated neighborhoods (block groups). The utilization of a validated audit tool used in another urban setting in Illinois helps to standardize an audit instrument that can be used in both large and small metropolitan areas. Further, the assessment of the availability of fruits and vegetables preferred by African-American and Latino communities is critical for long-term public health planning, and sustaining healthful dietary behaviors in these high risk groups if they continue to grow at the current rate in our study area. Our study, then, can serve as a baseline for subsequent studies as we work with local public health and city planning professionals to better serve African Americans and Latinos to improve dietary behaviors.

\section{ACKNOWLEDGEMENTS}

We thank Amy Kunkel, Gerald Charleston, Sarah Murphy and Bala Mutyala for assistance with data collection. This study was supported by a grant from the Illinois Council for Food and Agriculture Research (CFAR), and start-up funds from the Department of Kinesiology and Community Health at the University of Illinois at Urbana-Champaign. It was also partially supported by Robert Wood Johnson Foundation Healthy Eating and New Connections Grant \# 66952. The study was conducted as part of the STRONG (Synergistic Theory and Research on Obesity and Nutrition Group) Kids Project, which explores media effects on child obesity and health within family and community contexts. The instrument used for this study was adapted from the Southwest Chicago Food Store study (Zenk, SN, PI).

\section{REFERENCES}

[1] Grigsby-Toussaint, D.S., Zenk, S.N., Odoms-Young, A., Ruggiero, L. and Moise, I. (2010) Availability of commonly consumed and culturally specific fruits and vegetables in African-American and Latino neighborhoods. Journal of the American Dietetic Association, 110, 746752. doi:10.1016/j.jada.2010.02.008

[2] Zenk, S.N., Grigsby-Toussaint, D.S., Curry, S.J., Berbaum, M. and Schneider, L. (2010) Short-term temporal stability in observed retail food characteristics. Journal of Nutrition Education and Behavior, 42, 26-32. doi:10.1016/j.jneb.2009.01.005

[3] Larson, N.I., Story, M.T. and Nelson, M.C. (2009) Neighborhood environments: Disparities in access to healthy foods in the US. American Journal of Preventive Medicine, 36, 74-81. doi:10.1016/j.amepre.2008.09.025

[4] Morland, K., Wing, S. and Roux, A.D. (2002) The contextual effect of the local food environment on residents' diets: The atherosclerosis risk in communities study. American Journal of Public Health, 92, 1761-1767. doi:10.2105/AJPH.92.11.1761

[5] Odoms-Young, A.M., Zenk, S. and Mason, M. (2009) Measuring food availability and access in African-American communities: Implications for intervention and policy. American Journal of Preventive Medicine, 36, S145-S150. doi:10.1016/j.amepre.2009.01.001

[6] Ford, P.B. and Dzewaltowski, D.A. (2008) Disparities in obesity prevalence due to variation in the retail food environment: Three testable hypotheses. Nutrition Reviews, 66, 216-228. doi:10.1111/j.1753-4887.2008.00026.x

[7] Tessier, S., Traissac, P., Bricas, N., Maire, B., EymardDuvernay, S., El Ati, J. and Delpeuch, F. (2010) Food shopping transition: Socio-economic characteristics and motivations associated with use of supermarkets in a North African urban environment. Public Health Nutrition, 13, 1410-1418. doi:10.1017/S1368980010000601

[8] Johnson, C.M., Sharkey, J.R. and Dean, W.R. (2011) It's all about the children: A participant-driven photo-elicitation study of Mexican-origin mothers' food choices. BMC Women's Health, 11, 41. doi:10.1186/1472-6874-11-41

[9] Sealy, Y.M. (2010) Parents' food choices: Obesity among minority parents and children. Journal of Community Health Nursing, 27, 1-11. doi:10.1080/07370010903466072

[10] Yeh, M.C., Ickes, S.B., Lowenstein, L.M., Shuval, K., Ammerman, A.S., Farris, R. and Katz, D.L. (2008) Understanding barriers and facilitators of fruit and vegetable consumption among a diverse multi-ethnic population in the USA. Health Promotion International, 23, 42-51. doi:10.1093/heapro/dam044

[11] Ard, J.D., Fitzpatrick, S., Desmond, R.A., Sutton, B.S., Pisu, M., Allison, D.B., Franklin, F. and Baskin, M.L. (2007) The impact of cost on the availability of fruits and vegetables in the homes of schoolchildren in Birmingham, Alabama. American Journal of Public Health, 97, 367372. doi:10.2105/AJPH.2005.080655

[12] Morland, K. and Filomena, S. (2007) Disparities in the availability of fruits and vegetables between racially segregated urban neighbourhoods. Public Health Nutrition, 10, 1481-1489.

[13] Baker, E.A., Kelly, C., Barnidge, E., Strayhorn, J., Schootman, M., Struthers, J. and Griffith, D. (2006) The Garden of Eden: Acknowledging the impact of race and class in efforts to decrease obesity rates. American Journal of Public Health, 96, 1170-1174. doi:10.2105/AJPH.2004.049502

[14] Zenk, S.N., Schulz, A.J., Israel, B.A., James, S.A., Bao, S. and Wilson, M.L. (2006) Fruit and vegetable access differs by community racial composition and socioeconomic position in Detroit, Michigan. Ethnicity \& Disease, 16, 275-280

[15] Sharkey, J.R., Horel, S. and Dean, W.R. (2010) Neighborhood deprivation, vehicle ownership, and potential 
spatial access to a variety of fruits and vegetables in a large rural area in Texas. International Journal of Health Geographics, 9, 26. doi:10.1186/1476-072X-9-26

[16] Mather, M., Pollard, K. and Jacobsen, L.A. (2011) Reports on America: First results from the 2010 census. Population Reference Bureau, Washington DC.

[17] Bureau US. (2010) USA QuickFacts. US Census Bureau, Washington DC.

[18] Budget, O.M.B. (2000) Standards for defining metropolitan and metropolitan statistical areas: Notice. In: Budget, O.M.B., Ed., Federal Register, Office of Management and Budget, Washington DC, 1-12.

[19] Harrison, K., Bost, K.K., McBride, B.A., Donovan, S.M., Grigsby-Toussaint, D.S., Kim, J., Liechty, J.M., Wiley, A., Teran-Garcia, M. and Jacobsohn, G.C. (2011) Toward a developmental conceptualization of contributors to weight imbalance in childhood: The Six-Cs model. Child Development Perspectives, 5, 50-58. doi:10.1111/j.1750-8606.2010.00150.x

[20] Wakimoto, P., Block, G., Mandel, S. and Medina, N. (2006) Development and reliability of brief dietary assessment tools for Hispanics. Preventing Chronic Disease, 3, A95.

[21] Kittler, P. and Sucher, K. (2001) Culture in America: A nutrition handbook, Van Nostrand Reinhold, New York.

[22] Coates, J., Eley, G., Block, G., Gunther, E.W., Sowell,
A.L., Grossman, C. and Greenberg, R.S. (1991) An evaluation of a food frequency questionnaire for assessing dietary intake of specific carotenoids and vitamin $\mathrm{E}$ among low-income black women. American Journal of Epidemiology, 134, 658-671.

[23] Gustafson, A.A., Lewis, S., Wilson, C. and Jilcott-Pitts, S. (2012) Validation of food store environment secondary data source and the role of neighborhood deprivation in Appalachia, Kentucky. BMC Public Health, 12, 688. doi:10.1186/1471-2458-12-688

[24] Smith, D.M., Cummins, S., Taylor, M., Dawson, J., Marshall, D., Sparks, L. and Anderson, A.S. (2010) Neighbourhood food environment and area deprivation: Spatial accessibility to grocery stores selling fresh fruit and vegetables in urban and rural settings. International Journal of Epidemiology, 39, 277-284. doi:10.1093/ije/dyp221

[25] Lee, H. (2012) The role of local food availability in explaining obesity risk among young school-aged children. Social Science \& Medicine, 74, 1193-1203. doi:10.1016/j.socscimed.2011.12.036

[26] Feng, J., Glass, T.A., Curriero, F.C., Stewart, W.F. and Schwartz, B.S. (2010) The built environment and obesity: A systematic review of the epidemiologic evidence. Health \& Place, 16, 175-190. doi:10.1016/j.healthplace.2009.09.008 